\title{
A Hierarchical Comparison on Influence Paths from Cognitive \& Emotional Trust to Proactive Behavior Between China and Japan
}

\author{
Pei Liu \\ School of Management and Economics, North China \\ University of Water Resources and Electric Power \\ Zhengzhou, China \\ delicatemechanics@163.com
}

\author{
Zhen Li \\ Data Science Laboratory, RISS \\ Kansai University \\ Osaka, Japan \\ lzhen0205@gmail.com
}

\begin{abstract}
Little research examined how and why cognitive and emotional trust in supervisor influence employees' proactive behavior (PB). The following findings were obtained by applying a hierarchical cross-national comparison framework on influence path to Chinese and Japanese samples: 1) the confirmatory cross-national comparisons on higher-order level pathways confirmed that in both China and Japan, the influence of cognitive trust on $\mathrm{PB}$ is mediated by prospects of performance and growth, and the influence of emotional trust on $P B$ is mediated by warmth and happiness; 2) the exploratory crossnational comparison on lower-order level pathways revealed that the detailed structures of the two pathways were not much different between China and Japan, except in the depending willingness dimension of emotional trust; 3) finally, by comparing the findings of 1) and 2), we attempted to distinguish the inevitable parts and cultural peculiar parts within the two pathway.
\end{abstract}

Keywords-proactive behavior; cognitive \& emotioal trust; prospects of performance \& growth; warmth \& happiness; influence path

\section{INTRODUCTION}

In recent years, faced with the increasing uncertainty in occupations and organizations, employees' proactive behavior (PB), defined as the anticipatory actions that voluntarily change situations or themselves to cope with future threats and opportunities, becomes more and more important for both business and career [1]. As an extra-role and uncertain behavior, employees' $\mathrm{PB}$ is largely dependent on their trust in the supervisor. However, little is known about the influence processes. This paper aims to clarify the influence paths mediating the effects of the trust on PB.

\section{HYPOTHESES}

Literature on trust has almost reached a consensus that there are two basic elements in the definition of trust: (1) positive expectations: trustor's positive predictions such as "the trustee will make beneficial decisions and actions for me in widespread situations"; (2) willingness to be vulnerable: trustor' willingness to positively take the risks stemming from the interdependence with the trustee. According to distinct bases generating such expectations \& willingness, there are two kinds of trust influencing PB through different pathways.

\section{1. $C T \rightarrow P P G \rightarrow P B$ pathway}

Cognitive trust in supervisor (CT) is defined as the expectations \& willingness based on "the knowledge of supervisor's traits" [2, 3]. CT consists of three subdimensions: expectations based on the supervisor's 1) competence; 2) benevolence; 3) integrity [3]. The function of $\mathrm{CT}$ is to secure the productivity and healthiness of social exchange relationship (SER), by focusing on the outcome of exchange from the perspective of "gains and losses" [4]. For this reason, it is reasonable to think that (1) CT is deeply involved in the exchanges of the objective resources with general usefulness such as performance, growth, and rewards; and (2) CT is premised on the task \& management dependence of employees on the supervisor.

CT secures productivity and healthiness of SER by generating the predictions about what outcomes the reciprocal altruistic behavior will lead to. The process that PB brings about desirable performance \& growth is complex and uncertain, because it depends on a wide range of supervisor decisions and situational factors. According to [5], whether understand the whole picture is critical to forecast how the change in a condition causes the changes in the overall system (i.e., the structure of task \& management dependence in here). Therefore, we consider that insights about the whole picture, such as prospects of performance and growth (PPG), are essential in predicting such complex and uncertain process. PPG here refers to "how PB will influence my performance and growth in career, in what kind of process and with how much success ratio." Furthermore, to form PPG, it is necessary to systematically forecast the supervisor's widespread decisions under unspecified situations based on CT. Thus, through forming PPG, CT functions to steer PB towards the courses with a high success rate:

\section{H1: The influence of $C T$ on $P B$ is mediated by PPG.}

\section{2. $C T \rightarrow E T(\rightarrow W H) \rightarrow P B$ pathway}

Emotional trust in supervisor (ET) is defined as the expectations \& willingness rooted in "a high-quality long-term relationship with the supervisor" [2, 3]. Based on existing literature, we identified three sub-dimensions of ET, and validated them with a series of factor analysis evidence: 1) emotional linkage is rooted in sympathy; 2) identification, 
meaning the sense of fellowship due to the shared long-term interests and values, is rooted in perspective taking; 3) depending willingness, meaning a willingness to deepen the obligation in the relationship, is rooted in reciprocity [3].

The function of ET is to build up a base of the long-term relationship, by focusing on the process of exchange itself from the perspective of "maintaining SER". Hence, it is appropriate to consider that (1) ET is closely involved in the exchanges of the subjective resources with personal happiness; and (2) ET is premised on the interdependence in the happiness of work life between employees and the supervisor.

ET builds up the base of relationship by arousing altruistic needs to motivate the reciprocal altruistic behavior. The level of ET, by definition, mirrors the quality of a long-term relationship with the supervisor that can give employees warmth \& happiness (WH; [6]). The feeling of WH, which acts as a sensor to sense how good the relationship is, will arouse a group-oriented altruistic need such as "for the common long-term interests" to motivate PB. Thus, by stably bringing about WH, ET serves as a long-lasting power source for PB:

\section{H2-a: The influence of ET on PB is mediated by WH.}

And, it is reasonable to think that CT plays a role of controlling ET to be an adequate level [2]. For instance, employees are unlikely to share long-term interests with a supervisor without benevolence/integrity. Hence, we have:

\section{H2-b: The level of CT positively relates to the level of ET.}

\section{METHOD}

The Influence of Trust on Work-Related Attitudes and Behaviors (ITWAB) project is a continuing cross-national survey for theory construction and database creation. In the first stage of the project, the data was collected from Chinese and Japanese employees in 2013. The language expression between the questionnaire's Chinese and Japanese versions was checked by a linguist. All respondents answered the questionnaire via the specified websites.

Samples. Chinese employees' samples $(n=301)$ were gathered from seven cities of China: Shenzhen, Guiyang, Huainan, Tianjin, Shenyang, Anshan, and Jilin. 48.2\% of respondents were male and $51.8 \%$ were female. Their average age was 31.39 years $(S D=7.48)$, average job tenure was 6.25 years $(S D=7.31)$, and the average length of time having worked with the current supervisor was 4.43 years $(S D=5.02)$. In terms of education, $6.3 \%$ had high school or vocational degrees, $23.9 \%$ had junior college degrees, $39.9 \%$ had college degrees, and $29.9 \%$ had graduate degrees. In terms of post, $49.8 \%$ were general employees, $18.9 \%$ were low level managers, $17.3 \%$ were middle level managers, $6.3 \%$ were top level managers, and others (7.6\%).

Japanese employees' samples $(n=475)$ were collected from all over Japan through a research company. $54.7 \%$ of respondents were male and $45.3 \%$ were female. Their average age was 38.64 years $(S D=6.55)$, average job tenure was 12.90 years $(S D=7.72)$, and the average length of time having worked with the current supervisor was 4.31 years $(S D=4.76)$.
In terms of education, $25.5 \%$ had high school degrees, $22.7 \%$ had junior college or vocational degrees, $42.9 \%$ had college degrees, and $8.8 \%$ had graduate degrees. In terms of post, $44.8 \%$ were general employees, $27.2 \%$ and $22.1 \%$ were low and middle level managers, respectively, and others (5.9\%).

Measures. All variables were measured on a 7-point scale form $1=$ strongly disagree to $7=$ strongly agree.

CT was measured with a 14-item scale (CTS-S; Chinese $\alpha$ $=.97$, Japanese $\alpha=.97 ; 5$ items of competence, 4 items of benevolence, and 5 items of integrity), and ET was measured using a 14-item Scale (ETS-S; Chinese $\alpha=.96$, Japanese $\alpha$ $=.97 ; 5$ items of emotional linkage, 5 items of identification, and 4 items of depending willingness) which were developed in ITWAB project [3].

PPG was measured with 4 items (Chinese $\alpha=.89$, Japanese $\alpha=.90$ ). An example item was "Working with the current supervisor, I can develop my potential to achieve a desirable growth."

WH was measured using 3 items (Chinese $\alpha=.93$, Japanese $\alpha=.93$ ). An example item was "I feel very warm in this work environment."

PB was measured using a 12-item PBS developed in ITWAB project (Chinese $\alpha=.93$, Japanese $\alpha=.95 ; 3$ items of heroic, 3 items of self-advancement, 3 items of cooperation building, and 3 items of organization innovation) [1].

Using the two countries' data, this paper attempts to establish a hierarchical cross-national comparison framework consisting of three kinds of comparison: (1) the confirmatory cross-national comparison on higher-order level pathways; (2) the exploratory cross-national comparison on lower-order level pathways; (3) the inter-level comparison on a pathway.

\section{RESULTS}

\section{Measurement Models}

To test the validity of CT \& ET' conceptualizations, we confirmed the metric invariance of their measurement models across Chinese and Japanese samples. Figure 1 shows that the metric invariance model $(d f=22)$ met the criterion of CFI $\geq .95$ and $\mathrm{SRMR} \leq .08$, using joint estimation. And, compared with the configural invariance model having no cross-group equality constraints on the six factor loadings $(d f=16)$, the metric invariance model produced a significant increase in chi-square $\Delta \chi^{2}(\Delta d f)=11.578(6), p=.07$. Thus, the metric invariance of both the two measurement models holds. In addition, the six factor loadings separately estimated with Chinese and Japanese samples were reported in Table 1.

\section{The Higher-Order Level Pathways}

To test two hypothesized pathways, we built a higherorder level structural model to make a confirmatory crossnational comparison. As shown in Figure 2, the configural invariance model met the criterion of CFI $\geq .95$ and SRMR $\leq .08$, using joint estimation. Thus all hypotheses were supported by Chinese and Japanese samples. In addition, the path coefficients separately estimated with the two countries' samples were reported (See Table 1). 


\section{The Lower-Order Level $C T \rightarrow P P G \rightarrow P B$ Pathway}

To further investigate the $C T \rightarrow P P G \rightarrow P B$ pathway in details, we built a lower-order level structural model to make an exploratory cross-national comparison. Figure 3 shows that the configural invariance model met the criterion of $\mathrm{CFI} \geq .95$ and $\mathrm{SRMR} \leq .08$, using joint estimation. Thus, the $C T \rightarrow P P G$ $\rightarrow P B$ pathways between China and Japan were the same in

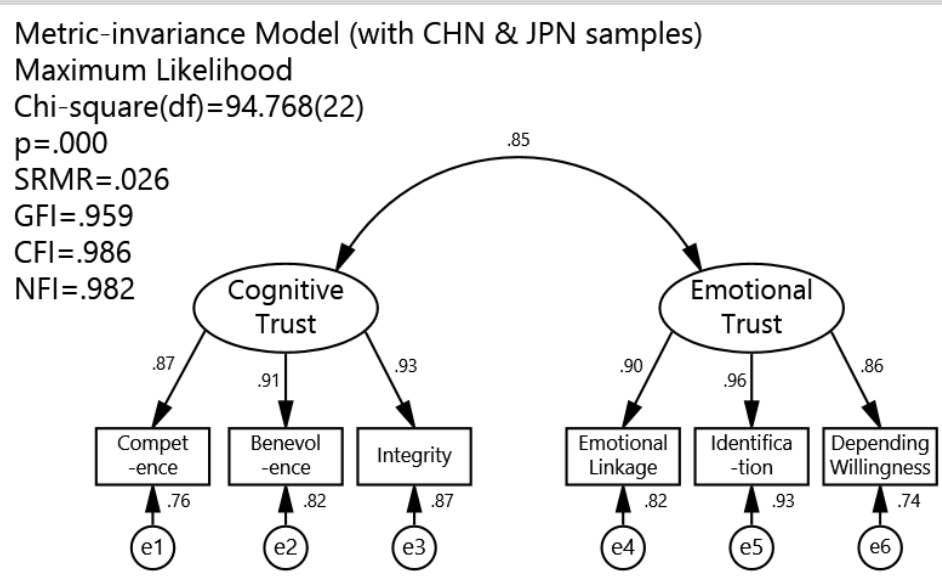

Fig. 1. Measurement models

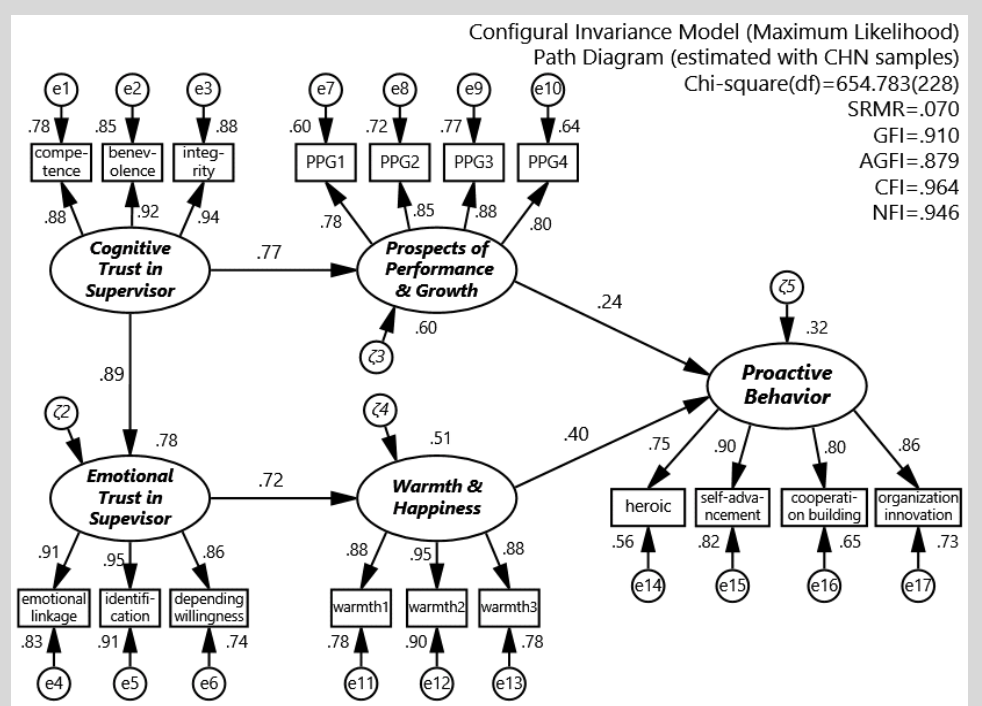

Fig. 2. Two higher-order level pathways

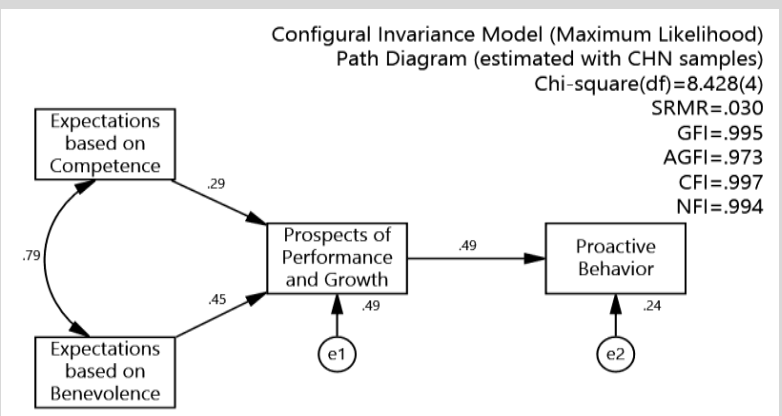

Fig. 3. Lower-order level CT $\rightarrow$ PPG $\rightarrow$ PB pathway
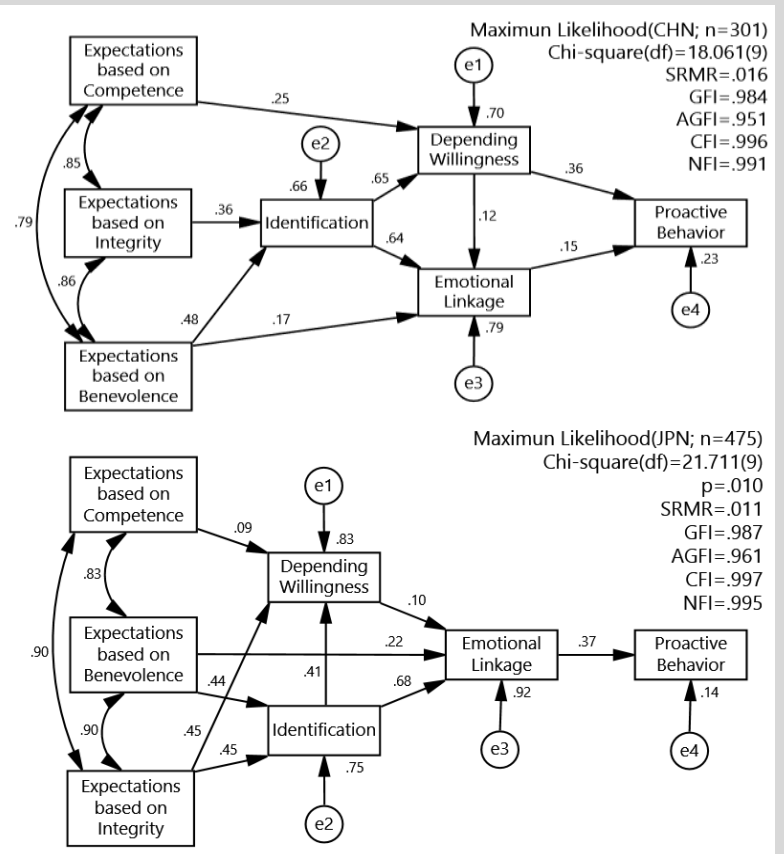

Fig. 4. Lower-order level $\mathrm{CT} \rightarrow \mathrm{ET} \rightarrow \mathrm{PB}$ pathway 
TABLE I. Separate Estimation: Standardized Path Coefficients

\begin{tabular}{lcc}
\hline & Chinese & Japanese \\
\hline Measurement models (Fig. 1) & & \\
CT - ET correlation & $.86^{* * *}$ & $.88^{* * *}$ \\
CT $\rightarrow$ Comptence path & $.88^{* * *}$ & $.89^{* * *}$ \\
CT $\rightarrow$ Benevolence path & $.92^{* * *}$ & $.93^{* * *}$ \\
CT $\rightarrow$ Integrity path & $.95^{* * *}$ & $.93^{* * *}$ \\
ET $\rightarrow$ Emotional linkage path & $.91^{* * *}$ & $.90^{* * *}$ \\
ET $\rightarrow$ Identification path & $.96^{* * *}$ & $.97^{* * *}$ \\
ET $\rightarrow$ Depending willingness path & $.85^{* * *}$ & $.88^{* * *}$ \\
\hline Higher-order level pathways (Fig. 2) & & \\
CT $\rightarrow$ PPG path (H1) & $.77^{* * *}$ & $.81^{* * *}$ \\
PPG $\rightarrow$ PB path (H1) & $.24^{* * *}$ & $.17^{* * *}$ \\
CT $\rightarrow$ ET path (H2-b) & $.89^{* * *}$ & $.89^{* * *}$ \\
ET $\rightarrow$ WH path (H2-a) & $.72^{* * *}$ & $.48^{* * *}$ \\
WH $\rightarrow$ PB path (H2-a) & $.40^{* * *}$ & $.35^{* * *}$ \\
\hline Lower-order level CT $\rightarrow$ PPG $\rightarrow$ PB pathway (Fig. 3) & \\
Comptence $\rightarrow$ PPG path & $.29^{* * *}$ & $.38^{* * *}$ \\
Benevolence $\rightarrow$ PPG path & $.45^{* * *}$ & $.37^{* * *}$ \\
Integrity $\rightarrow$ PPG path & $.09(p=.385)$ & $.06(p=.425)$ \\
PPG $\rightarrow$ PB path & $.49^{* * *}$ & $.30^{* * *}$ \\
\hline & & a. $* * * p<.001$. \\
\end{tabular}

the detailed structure. Also, the path coefficients were reported in Table 1.

\section{The Lower-Order Level $C T \rightarrow E T \rightarrow P B$ Pathway}

To investigate further the $C T \rightarrow E T \rightarrow P B$ pathway in details, we built two lower-order level structural models to make an exploratory cross-national comparison. To make it simple, we omitted the mediating variable WH. As a result of eliminating the non-significant paths, Figure 4 shows that the $C T \rightarrow E T \rightarrow P B$ pathways between China and Japan were different in the detailed structure. The path coefficients and model fit indices separately estimated with the two countries' samples were summarized in Figure 4 respectively.

\section{DISCUSSION}

\section{Validity and Generality of Measurement Models}

At the first, we examined whether the conceptualization of CT \& ET in ITWAB project has sufficient validity and generality. The metric invariance of the two measurement models was confirmed for Chinese and Japanese samples (Figure 1). This suggests that the two trusts are likely to have a general sub-dimensional structures and our conceptualization captures them well, at least for Chinese and Japanese employees.

\section{A Hierarchical Cross-National Comparison Framework on Influence Paths}

The purpose of this study is to investigate how and why CT \& ET affect employees' PB. We designed a hierarchical cross-national comparison framework to reveal the influence paths.

The two higher-order level pathways. As for the two higher-order level pathways, a confirmatory cross-national comparison summarized in Figure 2 confirmed the existence of a common mechanism underlying both Chinese and Japanese occupations/organizations.
The hypothesized structural model (Figure 2) enables us to grasp the overview of such social psychological mechanism. We expect the two hypothesized pathways can be confirmed in a wide range of countries.

Lower-order level $\mathrm{CT} \rightarrow \boldsymbol{P P G} \rightarrow \boldsymbol{P B}$ pathway. First, by the exploratory cross-national comparison, the configural invariance of the lower-order level model (Figure 3) clarified that the general mechanism's Chinese and Japanese varieties have many similarities in the aspect of task \& management dependence. The concrete findings summarized in Figure 3 are useful in the practice of international management.

Second, an inter-level comparison was made to distinguish the inevitable parts and cultural peculiar parts within the mechanism, especially the structure of task \& management dependence. In comparison with higher-order level $C T \rightarrow$ $P P G \rightarrow P B$ pathway (Figure 2), we speculate that competence $\rightarrow P P G$ path and $P P G \rightarrow P B$ path may reflect the inevitable parts. The two paths are consistent with [7]'s result that trustee's ability has positive effect on trustor's risk taking $(\beta$ $=.13, p<.05)$.

On the other hand, we speculate that benevolence $\rightarrow P P G$ path and integrity $\rightarrow P P G$ path may mirror the cultural peculiar parts. These paths are not consistent with [7]'s results that trustee's integrity $(\beta=.17, p<.05)$, not benevolence $(\beta=$ -.01), has positive effect on trustor's risk taking. If [7]'s data were majorly gathered from the U.S. and Europe, we can explain this as: (1) benevolence and integrity can functionally substitute for each other and; (2) in workplace, benevolence which is closely involved in dyadic relationships is more important for East Asians, whereas integrity which is involved in universal principles is more important for Americans and Europeans.

Lower-order level $\boldsymbol{C T} \rightarrow \boldsymbol{E T} \rightarrow \boldsymbol{P B}$ pathway. First, the findings of the exploratory cross-national comparison (Figure 4) revealed that in the aspect of work life happiness interdependence, the mechanism's Chinese and Japanese varieties are similar in emotional linkage and identification dimensions, but different in depending willingness dimension.

The distinction in Depending willingness $\rightarrow P B$ path suggests that Chinese and Japanese employees' major motives for PB are different. For Chinese employees, an important motive for engaging in PB is to make contribution to the longterm interests shared with the supervisor so that they can repay the obligation to him/her. In contrast, Japanese employees have not such motive for PB.

The distinction in Integrity $\rightarrow$ Depending willingness path implies that whereas Chinese employees are not willing to depend on a supervisor who has integrity but neither competence nor the shared long-term interests, Japanese employees can readily depend on him/her.

These distinctions in depending willingness dimension can be explained by the distinction in "strength of reciprocity norm" and "occupational/organizational mobility" between Chinese and Japanese varieties. 
Second, we made an inter-level comparison to distinguish the inevitable parts and cultural peculiar parts within the mechanism, especially the structure of work life happiness interdependence. Figure 4 shows that the influence paths involved with depending willingness, which is rooted in reciprocity, may reflect the cultural peculiar parts. On the other hand, the influence paths involved with emotional linkage and identification, which are rooted in sympathy and perspective taking respectively, may mirror the inevitable parts. This speculation is consistent with the studies of sympathy and perspective-taking [8].

In ITWAB project, we established a hierarchical crossnational comparison framework to analyze influence paths. The results of the confirmatory cross-national comparisons on higher-order level pathways are useful for theory construction on the underlying mechanism. The findings of the exploratory cross-national comparison on lower-order level pathways are useful to clarify how the mechanism's national varieties resemble/differ from each other in detail. And, the findings of the inter-level comparison on a pathway are useful in distinguishing the inevitable parts and cultural peculiar parts within the mechanism. Such understandings about the general mechanism and its national varieties can contribute to both theory building and the international management practice.

\section{REFERENCES}

[1] P. Liu, "The dimensional structure, scale of proactive behavior, and their comparisons between Japan and China: Item response theory and structural equation modeling approach," The Japanese Journal of Behaviormetrics, 42(2), 2015, in press.

[2] D. McAllister, "Affect- and cognition-based trust as foundations for interpersonal cooperation in organizations," Academy of Management Journal. 38, 1995, pp.24-59.

[3] P. Liu, "The dimensional structure and scale development of cognitiveand emotional-trust: Using Japanese and Chinese samples," Kobe University Graduate Student Working Paper No. 201305a., 2013. http://www.b.kobe-u.ac.jp/stuwp/2013/201305a.pdf

[4] P.M. Blau, Exchange and power in social life, New York: John Wiley, 1964.

[5] Yotaro Hatamura, Learning from design failures, Springer, 2009.

[6] A.C. Cuddy, M. Kohut, \& J. Neffinger, "Connect, then lead," Harvard Business Review, 91, 2013, pp.54-61.

[7] J.A. Colquitt, B.A. Scott, \& J.A. LePine, "Trust, trustworthiness, and trust propensity: A meta-analytic test of their unique relationships with risk taking and job performance," Journal of Applied Psychology, 92, 2007,pp. 909-927.

[8] A. Grant, "In the Company of Givers and Takers," Harvard Buisness Review, 91, 2013, pp.90-97. 